accounting for over $90 \%$ of these cases. Two-thirds of patients received bystander $\mathrm{CPR}$ and a defibrillator was attached to the patient in $14.9 \%(n=150)$ of OHCAs before arrival of emergency medical services with a shock being ultimately delivered in 3.9\% $(n=39)$ of cases. Overall survival to hospital discharge for the cohort was $6.0 \%(n=60)$ and no significant change in survival was noted over the 6-year study period. The rate of survival was dependent on OHCA aetiology, (figure 1). Independent predictors of survival to hospital discharge included bystander witnessed OHCA, a shockable initial rhythm and a bystander defibrillation attempt.

Conclusions The high prevalence of non-medical OHCAs and the OHCA location need to be considered when developing OHCA care pathways and preventative strategies to reduce the burden of OHCAs in young adults.

\section{HYPERTENSION IN THE ACUTE MEDICAL ASSESSMENT UNIT IN ST. LUKE'S GENERAL HOSPITAL KILKENNY - ARE WE ADHERING TO GUIDELINES?}

K Millar, C Crowley, P Cotter. St Luke's Hospital, Kilkenny, Ireland

10.1136/heartjnl-2020-ICS.53

Background Hypertension is a common reason for GP referral to the Acute Medical Assessment Unit (AMAU) in St. Luke's Hospital, Kilkenny (SLK). Hypertensive emergency requires urgent and aggressive blood pressure control to limit endorgan damage. Management of hypertension has been proven to reduce the incidence of cardiac events, cardiovascular death and stroke. The aims of this audit were: 1. To ascertain what percentage of patients referred with hypertension were diagnosed with hypertensive emergency. 2. To review whether or not patients referred with hypertension had had appropriate work up (with electrocardiogram and urine dipstick.) 3. To review what percentage of patients deemed not to have hypertensive emergency received unindicated stat doses of antihypertensives. 4. To assess what percentage of patients were managed in compliance with the ESC-ESH Hypertension Guidelines 2018 on discharge.

Methods We carried out a retrospective review of the case notes and discharge letters of 106 patients who were referred to the AMAU by GPs with hypertension between January 2019 and January 2020.

Results 91 patients were included in the study. 35 (38\%) were male and $56(62 \%)$ were female. The median age of patients was 60.2 patients $(2.2 \%)$ were diagnosed with hypertensive emergency. Both were managed with oral antihypertensives. 83 patients (91\%) were asked about symptoms of hypertension, 86 patients (95\%) had an ECG. Only 26 (29\%) had a urine dipstick. Of the 89 patients deemed not to have hypertensive emergency, $43(48 \%)$ received a stat dose of an oral antihypertensive. 11 patients $(12 \%)$ had their stage of hypertension documented. Of these, 8 (73\%) were discharged on an appropriate antihypertensives. 24 patients $(27 \%)$ had stage 1 hypertension, $27(30 \%)$ had stage 2 and 38 (43\%) had stage 3. On discharge, 40 patients (45\%) were discharged on dual/triple antihypertensive regimens (in accordance with ESC-ESH guidelines.)

Of the 89 patients discharged from the AMAU, 69 (78\%) were booked for a 24 hour ABPM and 26 (29\%) were referred for TTE.
Conclusion This audit highlights numerous areas for improvement in the management of patients presenting to the AMAU in SLK with hypertension. It revealed that almost half of patients deemed to have no evidence of malignant hypertension/end-organ damage requiring immediate blood pressure control received at least one stat dose of an anti-hypertensive. The staging and management of hypertension remains a confusing area for some NCHDs, evidenced by the fact that only $12 \%$ of patients had their hypertension staged during their review. Positively, almost $80 \%$ of patients who presented to the AMAU with hypertension were booked for an outpatient 24 hour ABPM, however only 29\% were booked for a TTE. We aim to develop a proforma for managing patients presenting with hypertension and re-educate our staff on the investigation and management of hypertensive patients in order to closer align our practices with ESC-ESH guidelines.

\section{RESIDUAL RISK IN CARDIAC REHAB: CAN WE REDUCE- IT MORE? ELIGIBILITY FOR ICOSAPENT ETHYL IN PATIENTS ATTENDING CARDIAC REHABILITATION}

${ }^{1} \mathrm{~S}$ Gaine, ${ }^{2} \mathrm{JJ}$ Coughlan, ${ }^{3} \mathrm{~V}$ Maher, ${ }^{3} \mathrm{M}$ Waters. 'St James's Hospital, Dublin, Ireland; ${ }^{2}$ German Heart Centre, Munich, Germany; ${ }^{3}$ Tallaght University Hospital, Tallaght, Dublin, Ireland

\subsection{6/heartjnl-2020-ICS.54}

Introduction Patients with elevated triglycerides (TG) are at increased risk for ischemic events despite statin therapy and controlled low-density lipoprotein cholesterol (LDL-C). The REDUCE IT trial showed that a highly purified Eicosapentaenoic acid (EPA) ester, Icosapent Ethyl or Ethyl Eicosapentaenoic acid (E-EPA), reduces the risk of ischemic events and cardiovascular (CV) death in patients with elevated TG levels despite statin therapy. The NNT for the first occurrence of major adverse cardiac events for the five-point primary composite endpoint was 21. E-EPA is also a dominant strategy from a cost-effectiveness perspective in the study. Thus, the 2019 ESC/EAS guidelines recommend E-EPA for patients with persistently raised TGs despite treatment with a statin. Our aim was to assess the proportion of patients attending cardiac rehabilitation who may benefit from E-EPA therapy as per REDUCE-IT trial criteria and the 2019 ESC/EAS guidelines.

Methods We prospectively collected data on all cardiac rehabilitation patients in our centre in 2018/2019. We then performed a hierarchal analysis of these patients to determine the percentage of patients post MI/CABG that would meet criteria for E-EPA as per REDUCE-IT trial criteria and ESC/EAS guidelines. $=$

The REDUCE IT trial criteria were:

- $\geq 45$ years and established ASCVD

- 50 years with DM and at least one other CV RF

- Fasting TG level of 1.69 to $5.63 \mathrm{mmol} / \mathrm{L}$

- LDL-C level of 1.06 to $2.59 \mathrm{mmol} / \mathrm{L}$

- Receiving a stable dose of a statin for at least 4 weeks

The initial trial protocol enrolled patients with a TG level as low as $1.52 \mathrm{mmol} / \mathrm{L}$ to account for the $\sim 10 \%$ variability in TG levels. This protocol was amended and changed the lower level for TG to $2.26 \mathrm{mmol} / \mathrm{L}$. We analysed patients as per both protocols. 